\title{
Heritage Corridor Narrative Space System Research Case on the Ancient Lancang- Mojiang Tea Horse Road
}

\author{
Hui LI \\ College of Urban Construction and Administration \\ Yunnan University \\ Kunming, China \\ e-mail: ydlihui@qq.com \\ Min GAO \\ College of Urban Construction and Administration \\ Yunnan University \\ Kunming, China \\ e-mail:gaomin@qq.com \\ Wenwen TAN \\ College of Urban Construction and Administration \\ Yunnan University \\ Kunming, China \\ e-mail: tanwenwen@qq.com
}

\author{
Pan LI \\ College of Urban Construction and Administration \\ Yunnan University \\ Kunming, China \\ e-mail: lipan@qq.com \\ Guoyan LI \\ College of Urban Construction and Administration \\ Yunnan University \\ Kunming, China \\ e-mail: kmguoyanli@126.com \\ Weijing WANG \\ College of Urban Construction and Administration \\ Yunnan University \\ Kunming, China \\ e-mail: wangweijing@qq.com
}

\begin{abstract}
The Lancang-Mojiang ancient Tea Horse Road is an important branch of the Puer Ancient Tea Horse Road. With the development of urbanization, the Tea Horse Road and its spatial have been changed dramatically, and the cultural heritage along the road faced the threat of gradually disappear. This article adopts the method of narrative with "The Lancang-Mojiang ancient Tea Horse Road" as an example to explore narrative space structure of the corridor. It has shown that The Tea Horse Road narrative space structure presents triaxial four-core type. Narrative elements formed development main axis relying on Lancang to Mojiang main corridor, and Lancang, Simao region two secondary vertical development axis, four-core respectively is refers to the four regions, namely Lancang river basin in Simao and Lancang border as well as in Simao, Ninger, Mojiang County as the center. The microscopic analyses of the narrative space structure of the corridor not only obtained the narrative space structure of historic urban area, but also dig out the deep connotation of all kinds of narrative elements. It will provide reference basis for the protection of the historic urban area.
\end{abstract}

Keywords-ancient tea horse road; narratology; narrative space structure

\section{INTRODUCTION}

This article main research: study Ancient LancangMojiang Tea Horse Road historical stages evolution, preliminary understanding the evolution of spatial patterns in different historical periods; the study of narrative elements of the corridor, mainly from the natural and artificial, labor, events, four types of narrative elements, comprehensive mining corridor resource information; the narrative space structure of the corridor research.

The United States is the earliest country presenting the concept of heritage corridor and heritage area. In 1984 congress established the first line of the United States and the world heritage corridor, Illinois and Michigan canal national heritage corridor. It marks the heritage corridor concept was proposed and established, and the heritage corridor bring into the scope of legal protection [1]. At present, the existing 10 heritage corridor and 27 heritage area formally approved by the United States congress

Other countries also exist with heritage corridor similar cultural route and heritage line concept, such as the San Diego DE hole post pilgrimage route, Anda Luce heritage line, pirates line, Jewish tourist route and European Roman art line etc. Foreign study of heritage corridor is mainly focused on produce of the concept, the judgment standard, the resources mining, etc., in terms of use, most research focuses on the heritage corridor in the protection of cultural heritage and to grasp overall strategy.

About the protection and research of heritage corridor is still in its infancy in China. In 2001 by Beijing university city and the environment department Zhifang Wang, published his paper "heritage corridor, a new heritage protection method", the concept of heritage corridor first time importing to domestic, until 2006 people begin to realize the heritage corridor importance of heritage conservation areas in China, the related research began to more.

Research focuses on the following five aspects: review of heritage corridor and foreign heritage corridor [2-3]; with heritage corridor model to promote heritage protection 
research [4]; with heritage corridor pattern to stimulate tourism economy [5]; the study of heritage corridor building [6]; special research of several cases. Heritage corridor connotation is rich and has the huge system, characteristics of regional, comprehensive, living condition, currently on a world scale, the lack of systematic and integral space research for the heritage corridor resources.

In recent 10 years, the study of narrative environment gradually developed into the study of cognitive narrative and space narrative, and then focuses on the application of urban spatial analysis and organization.

\section{RESEARCH METHODS}

\section{A. Literature Survey Method}

Through literature review analysis related research status of narrative space systems and heritage corridor. Collect and analysis related document literature of geographical history culture, national culture and non-material culture along the Lancang Mojiang ancient tea-horse road.

\section{B. Field Research Method}

Through on-the-spot investigation determines the distribution and save status of the cultural heritage resources along the Lancang Mojiang ancient tea-horse road.

\section{Map Superposition Method}

Using ArcGIS software fall to all kinds of narrative elements respectively on the map. For artificial and events elements with time, draw respectively it to the map of the period, using the map superposition method after analysis, it can be seen that the evolution of spatial structure of the two types of elements.

\section{Comprehensive Analysis Methods}

This study combined with narrative and humanistic geography, city planning, heritage protection, archaeological historical relics, ecology theory and relevant theories and methods, using multi-angle and comprehensive analysis method, in-depth analysis of the heritage corridor Ancient Lancang-Mojiang Tea-Horse Road.

\section{GENERAL SITUATION OF RESEARCH REGION}

The study area is located between north latitude $22^{\circ} 01$ '$23^{\circ} 59^{\prime}$, longitude $99^{\circ} 29^{\prime}-102^{\circ} 04^{\prime}$, from west to east across the Puer region, the tropic of cancer across the border. Area have 700 hectares ancient tea mountain scene, the Tiger Jumped Stone wonders, Simao Plum Lake etc superior natural conditions, the along the Lancang river area is center of world's tea country of origin, a large number of ancient tea tree and tea garden has laid a foundation to formation and development of Ancient Tea Horse Road. In addition, Blang nationality, Yi nationality, Hani localized in the area and $\mathrm{Wa}$, Dai, Lahu and other foreign national fusion together has formed the colorful ethnic culture and religious culture. In the period of the trade thriving tea and horse, Ancient Puer Tea Horse Road with Simao, Ninger area as the center has radiated to the surrounding, from Tea Horse North Road, Tea Horse South Road, Tea Horse West Road, and Tea Horse East Road to Mainland China, Tibet,
India, Thailand and Burma. This shows the study area have typical and representative.

\section{NARRATIVE SPACE FACTORS OF THE RESEARCH REGION}

\section{A. Natural Elements}

Lancang County territory is gentle slope more than steep slope, while in Simao, Ninger, Mojiang County is steep slope more than gentle slope in the research area. Within the territory have mountains of Ailaoshan, Wuliangshan, Daheishan, Ximengshan Mountain, etc., from north to south. Many mountains gestate rich animal and plant resources, and the world rare sunbird, long tail turtles and other rare animals can be found in the dense forest in the mountains. Water system has many tributaries of the Honghe River, the Lancang River, Nujiang River, and three rivers such as the Babianjiang, Xiaohei River, South Island River, Simao River, etc.

\section{B. Humanistic Elements}

Puer has a more than 40 ethnic minorities, Lancang County is the only Lahu autonomous county, Mojiang County is the only of the Hani nationality autonomous county, Ancient Lancang-Mojiang Tea Horse Road along the various ethnic minority clothing because of its unique customs and etiquette, diet, literature and art, and characteristics residential building has added bright beautiful color for road. Along with the foreign Buddhism and religion infiltration, on the basis of local culture and integrated into a foreign culture, make along the ancient road culture more enrichment and diversification.

In analysis, you can see that Ancient Lancang-Mojiang Tea Horse Road narrative elements have great history, art, science and technology and social value, and the nature, the humanities, artificial, event narrative elements are exist side by side and play a part together and closely linked.

\section{Construct the NARRATIVE Space SyStem IN THE RESEARCH REGION}

The natural elements have formed after a long period the accumulation of precipitation of Geology and geomorphology change, therefore not to study natural elements by stages. Along research area the ethnic minority distribution in Puer is a number of large dispersed and small gather together, and distributed characteristics is dispersed and widely, difficult to accurately show its location, so no analyze spatial structure of the human elements. Emergence and happen of artificial and events elements are in a particular period of the ancient road development. First research on these two types of elements by stages, then using the overlay analysis with map superposition method, can clearly to show the evolution and development of the material elements in ancient road spatial pattern. Finally the superposition of natural, artificial and events three types' elements can research and summarize corridor space evolution. 


\section{A. Spatial Structure Analysis of the Natural Elements}

Puer city has rich and peculiar natural resources, also brought to along the Ancient Lancang-Mojiang Tea Horse Road many beautiful natural scenery, the vast majority of resources is adjacent to the road line distribution, mainly concentrated in the central area of the study area.

\section{B. Analysis of Spatial Structure of Artificial Factors}

Artificial elements are that people have created, due to the need of production and living or national religion and other cultural influences in a particular period, its distribution situation embodies the track changes of human activities. Before the Tang dynasty range of people is small, artificial factors are none on the space composition system. The distribution of material heritage is not concentrated, is relatively fragmented, but $80 \%$ of heritage point distributed along the road in Song and Yuan period, thus it can be seen that human activities is more frequent along the ancient road area in this period.

The Qing dynasty and the period of the Republic China is a peak period of development for Ancient Lancang Mojiang Tea Horse Road. Ancient Road line as axis, formed in Simao city, Ninger city, Mojiang city as the center of the three main nodes, as well as junction of Simao, Lancang in lancang river basin as the a secondary nodes. Space layout of the artificial factor during this period laid the basis of the spatial of the artificial elements structure in Ancient Lancang Mojiang Tea Horse Road. During this period material elements achieved more than 60 .

After 1949, Ancient Tea Horse Road was gradually replaced by modern roads, railways and other new traffic, gradually withdrew from the historical stage, artificial factors sharply reduce along the ancient road.

Overall, Ancient Lancang-Mojiang Tea Horse Road artificial elements present spatial layout of three axis and four nodes type, four nodes respectively means Lancang river basin of Lancang and Simao border, and area of Simao County, Ninger County and Mojiang County as the center.

\section{Analysis of Spatial Structure of Event Factors Elements}

Ancient Lanmo Tea Horse Road as the prosperous trade channel in the old days has happened many great events. According check of the historical data, before the Ming dynasty about record of Ancient Lanmo Tea Horse Road event is less, and currently accounts have more political events. After the Qing dynasty, famous Puer Tea make Puer area world-famous, along the Ancient Lanmo Tea Horse Road all kinds of events increase gradually, formed in Simao city, Ninger city and Mojiang city three nodes, and the event of Lancang region is still less. After 1949, the Ancient Road has declined, along road the important events has decreased, formed the scattered point distribution pattern.

Also using the map superposition method, has overlaid the event elements space structure map of the several historical period, then has get the diachronic spatial gray level structure map. According to the gray overlay chart, has get the space structure of Ancient Lanmo Tea Horse Road event elements of, which present space layout of two axis and three nodes type, formed in Simao city, Ninger city and Mojiang city three nodes, secondary axis is Lancang County north-south direction axis.

Using map superposition method to all kinds of elements can get collection of natural and artificial elements and events along Ancient Lanmo Tea horse Road. Analyzing narrative elements in gray overlay chart can clearly see, the heavier color area after superposition that show the area of the element collection greater amount, and it is an important node in the development of the ancient road.

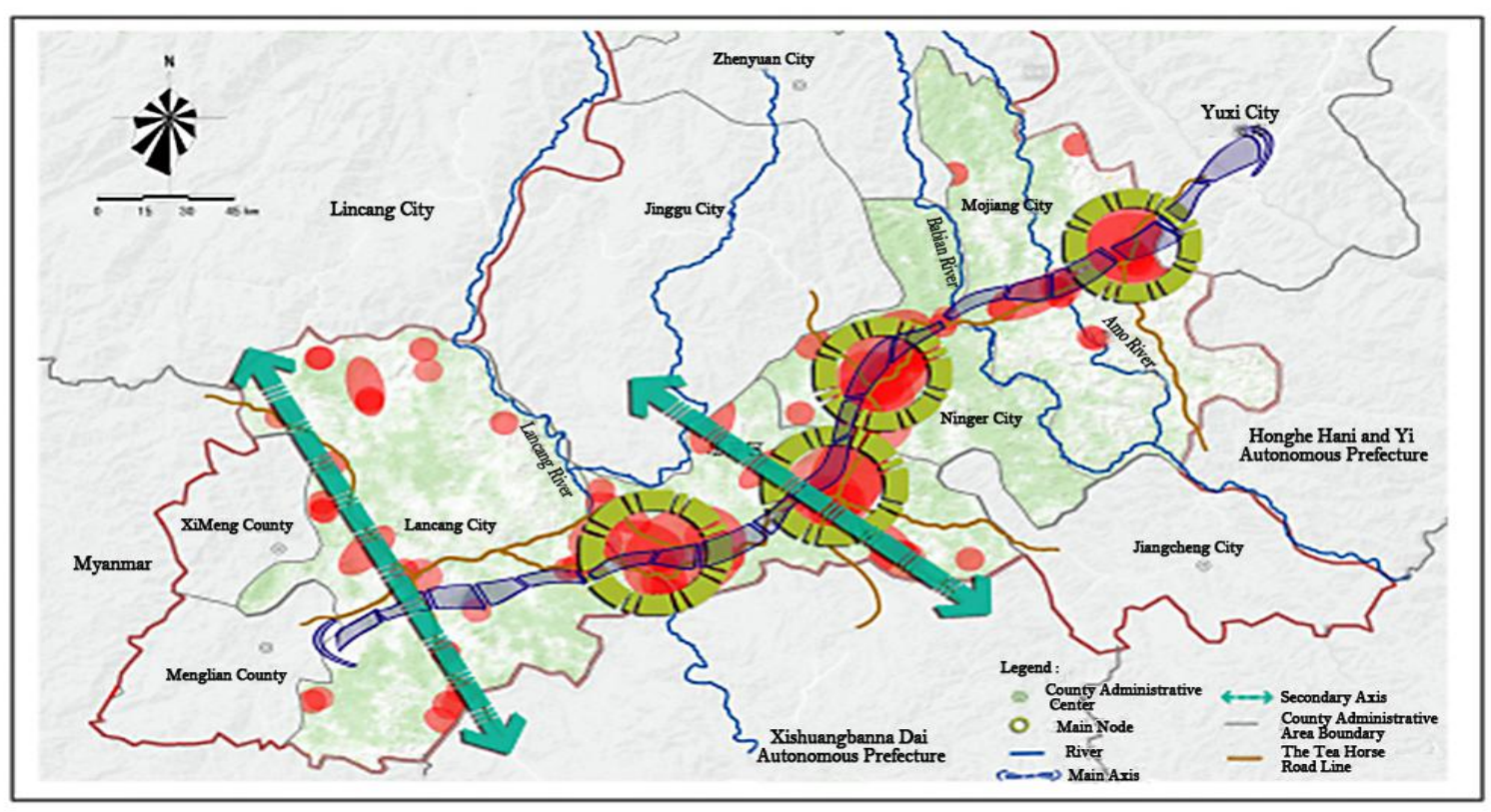

Figure 1. Narrative Space Structure of ancient Lancang-Mojiang tea horse road 
According to the gray degree map superposition color can be divided into three levels: color the heaviest area is grade I, it focuses more heritage resources, and the most shallow color area is grade III. On this basis, the corridor protection can be divided into three levels: level I and level II area as the core area, level III area as the construction control area, and the construction control areas expand outwardly certain areas as peripheral coordination area, general delineated with ancient road through the mountains, water and other boundaries.

Narrative space structure of rings of Ancient Lanmo Tea Horse Road is tree axis and four core type, the principal axis is development axis of Lancang to Mojiang based on the main road corridor, second axis are the two lines of Lancang and Simao region longitudinal development, four core refers to Lancang river basin of Simao and Lancang border land, Ninger, and Mojiang County as the center the four regions, as Figure 1.

\section{Sustainable Development Measure of Heritage Corridor}

On the basis of the above narrative space structure analyses, stainable development measure of heritage corridor has been put toward.

First, it is overall level protection of the Ancient Tea Horse Road heritage corridor. The protection of the core area is most strictly, adhere to the original true and original whole of the heritage, it is strictly prohibited any development or modification in the area; the key reserve protection mode is given way of to protect priority and development complementary; peripheral coordination area are mainly composed heritage tourism development.

Second, it is protection of the important node. Key nodes is the area with the most abundant historical resources in the corridor development process, its protection should show local characteristics and national culture diversity.

For example, the node of Lancang river valley is a concentration area of neolithic site that have very important value for archaeological research, at the same time the peculiar landform of Lancang river basin also created some famous historic sites, such as the cliff stone carving of Zhengkongjiang river, the first port of Simao Simaogang, stone carving have recorded the historical events occurred, Lancang river basin node is also one of the important node that show Ancient Lanmo Tea Horse Road feature. Ninger node is best Tea Horse Road preserved area within the territory of Puer, can be used it as ancient road protection demonstration area, and by showing and explanation advertise ancient historical and cultural connotation.

Third, it is protection of the narrative elements resources. Corridor resources excavated from narrative angle posses unique and uniqueness, and the protection should follow the principle of heritage protection: the original true, the original integrity, primitiveness. Should not be transformation and reconstruction in the process of protection and restoration of heritage resources, should be the old as before and to save its true.

Especially for ancient road reality, on the whole way of protection is save; For areas destroyed and difficult to repair that can set up a museum or site for absolute protection and it is strictly prohibited for road renovation or reconstruction.

Material heritage protection should not only for construction, tower, temple and other monomers, also should protect the original true around environment. For non-material heritage characteristic concentrated regions shall establish national traditional culture protection area. You can also set up a national intangible heritage inheritance learning institutions, at the same time, promotion and display is also indispensable for the features of ethnic culture and diversity in the corridor event.

\section{CONCLUSION AND DISCUSSION}

First, combing the historical context, it facilitates analysis of the diachronic research of ancient road. The development of ancient road has divided into four stages based on the characteristics of its history, which conducive to further concluded that space evolution pattern formed by the different historical period.

Second, it is the narrative elements resources research and overview. Four elements formed nature, human and human, events, which have listed the listing of heritage corridor narrative elements in Ancient Lanmo Tea Horse Road, a total of 234, in addition to the cultural elements, among them, 20 natural elements, 134 human factors, 80 factors of events.

Third, it is the spatial pattern research of the corridor. Narrative space structure that present a three axis and four cores.

Fourth, this study is only the initial stage of corridor space narrative structure analysis, future also need more indepth mining to each kind of narrative elements.

\section{ACKNOWLEDGMENT}

The authors would like to express appreciations to support by the National Natural Foundation of China (No. 51468064). The authors would like to express appreciations to colleagues in our laboratory for their valuable comments and other helps.

\section{REFERENCES}

[1] Asakawa, S., Yoshida, K. \& Yabe, K., "Perceptions of urban stream corridors within the greenway system of Sapporo". Landscape and Urban Planning. vol. 68(2-3), pp.167-182, 2004.

[2] Xiao Wang, Baichao Chen, "Conservation of the national heritage corridor in the United States: in the case of Blackstone River Valley," World Architecture. vol. 7, pp. 124-126, 2007.

[3] Xuesong Xi, Konglian Yu, Hailong Li, “ The Review of American national heritage management plan," Urban Planning International. Vol. 4, pp. 91-98, 2009.

[4] Yanan Wang, Xiaojia Zhang, Manqing Lu, "Study on planning of urban space system based construction of heritage corridor," Chinese Landscape Architecture. Vol. 12, pp85-87, 2010.

[5] Long Lu, Zhenfang Huang, "Establishment and application of indexes of qualitation evaluation of tourism value of the Grand canal," China Population Resources and Environment. Vol. 6, pp. 99-104, 2007.

[6] Dashan Qiao, Bing Feng, Huimin Zhai, "A preliminary discussion about the new approach of protecting and planning Guilin heritageconstructing Lijiang heritage corridor," Tourism Tribune. Vol. 22(11), pp. 28-31, 2007. 